\title{
The effect of ulinastatin on hemostasis in major orthopedic surgery
}

\author{
Jin Young Lee, Ji Young Lee, Jin Young Chon, Ho Sik Moon, and Sung Jin Hong \\ Department of Anesthesiology and Pain Medicine, The Catholic University of Korea College of Medicine, Seoul, Korea
}

Background: Ulinastatin, a urinary trypsin inhibitor, is widely used to treat acute systemic inflammatory disorders. However, the effects of ulinastatin, especially on the potential for hemostasis, have not been fully elucidated. This study examined whether ulinastatin had any beneficial effects on blood loss and blood transfusion requirements in patients undergoing major orthopedic surgery. Methods: Eighty patients, aged 18 to 75 years, scheduled for major orthopedic surgery were enrolled in this study and were divided into the ulinastatin $(n=40)$ and control ( $n=40$ ) groups. Following the induction of general anesthesia, and immediately before the surgical incision, the patients in the ulinastatin group were given 5,000 units/kg of ulinastatin, which were mixed in $100 \mathrm{ml}$ normal saline intravenously over $30 \mathrm{~min}$, while those in the control group received the same volume of normal saline. The amounts of blood loss, infused fluid, and transfused blood products were measured throughout the study period. Blood samples for coagulation parameters were obtained before inducing anesthesia (T1), at the end of surgery (T2), and $12 \mathrm{~h}$ after surgery (T3). Results: The amounts of blood loss and infused fluid during surgery were not significantly different between the two groups. However, $12 \mathrm{~h}$ postoperative blood loss was significantly less in the ulinastatin group than in the control group $(255.0 \pm 133.2 \mathrm{ml} \mathrm{VS} .395 .4 \pm 338.4 \mathrm{ml}, \mathrm{P}<0.05)$. Conclusions: Our data suggest that a single infusion of ulinastatin in major orthopedic surgery is associated with decreased blood loss in the early postoperative period. (Korean J Anesthesiol 2010; 58: 25 30)

\section{Key Words: Blood loss, Orthopedic surgery, Transfusion, Ulinastatin.}

\section{Introduction}

Despite advances in anesthesia and surgical care, some types of procedures associated with osteotomy during major orthopedic surgery can increase bleeding [1]. The blood vessels in bone are non-collapsible structures and will remain open when the bone is cut [1]. The exposed bony surfaces are not amenable to standard hemostatic maneuvers used during soft tissue surgery, which contributes to perioperative

Received: August 28, 2009. Revised: 1st, September 7, 2009; 2nd, October 21, 2009. Accepted: November 20, 2009.

Corresponding author: Sung Jin Hong, M.D., Department of Anesthesiology and Pain Medicine, The Catholic University of Korea College of Medicine, 62 Yeouido-dong, Yeongdeungpo-gu, Seoul 150-713, Korea. Tel: 82-2-3779-1097, Fax: 82-2-783-0368, E-mail: hongs@catholic.ac.kr (c) This is an open-access article distributed under the terms of the Creative Commons Attribution Non-Commercial License (http:// creativecommons.org/licenses/by-nc/3.0/), which permits unrestricted non-commercial use, distribution, and reproduction in any medium, provided the original work is properly cited. 
hemorrhage and activates coagulation and the fibrinolytic cascade $[2,3]$. Surgical manipulation and associated tissue trauma also contribute to the activation of tissue factors and the systemic inflammatory response [4]. The inflammatory process promotes the release of serine protease by neutrophils, macrophages, lymphocytes, and endothelial cells. Elastase, one of the proteases produced by neutrophils, is involved in these processes in a variety of ways. In animal models, increased elastase activity induces a disturbance of blood coagulation, leading to hypocoagulability [5]. Ulinastatin attenuates the elevation of neutrophil elastase release, thereby blunting the rise of pro-inflammatory cytokine levels; however, the actual mechanism in vivo is not clear $[6,7]$. Okida et al. [8] reported that ulinastatin normalized the coagulation function and prevented changes in TEG measurement during liver resection. Porte et al. [9] demonstrated that the success of antifibrinolytics in reducing perioperative bleeding suggests that hyperfibrinolysis is a major contributor to the bleeding diathesis. A recent clinical trial suggested that ulinastatin could inhibit coagulation and fibrinolysis in abdominal surgery [10]. Considering this study, we hypothesized that suppression of this acute phase reactive substance may reduce perioperative blood loss by improving hemostasis. In the present study, we measured a coagulation profile including antithrombin III (AT III) and fibrin degradation product (FDP) to evaluate the impact of ulinastatin on the coagulation system. AT III and FDP are useful markers for the diagnosis of altered hemostasis [7]. FDP is the most widely used fibrin-related marker by clinical laboratories [7]. On activation of the fibrinolytic cascade, plasmin catalyzes fibrin to FDP, increasing FDP levels. The natural anticoagulant, AT III, regulates the removal of thrombi from the vascular system [11]. The purpose of this study was to evaluate whether ulinastatin affected perioperative blood loss and blood transfusion requirements in patients undergoing major orthopedic surgery. We also evaluated the influence of ulinastatin on coagulation parameters.

\section{Materials and Methods}

After getting approval to conduct the study from the Institutional Review Board of the University Hospital, we enrolled 80 American Society of Anesthesiologists physical status I-III inpatients, aged 18 to 75 years, undergoing major orthopedic surgery, with an expected blood loss of more than $10 \%$ of their total estimated blood volume intraoperatively, including spinal fusion, total hip arthroplasty, unilateral intramedullary nailing of the femur, or fixation of pelvic bone fractures. Written informed consent was obtained from each participant. Exclusion criteria were patients with preoperative bleeding and clotting disorders, an abnormal coagulation test, thrombocytopenia, and severe renal, hepatic, or heart disease. Aspirin and other antiplatelet agents were discontinued 7 days before the scheduled procedure. The patients were divided into two groups in a controlled trial: 40 patients were given ulinastatin (ulinastatin group) and 40 patients were given the same volume of normal saline (control group).

\section{Interventions}

All patients were given an intramuscular injection of glycopyrrolate $0.2 \mathrm{mg}$ and midazolam $0.05 \mathrm{mg} / \mathrm{kg} 15$ min preoperatively. Before the induction of anesthesia, a 22-gauge catheter was inserted into a radial artery connected to a pressure transducer to measure blood pressure and to collect blood samples. Standard monitoring (Intellivue MP70 Anesthesia, Philips, USA), including electrocardiography, pulse oximetry, capnography, noninvasive arterial blood pressure, and body temperature, was performed throughout the procedure. Anesthesia was induced with intravenous propofol 2-3 mg/kg and fentanyl $1.5 \mu \mathrm{g} / \mathrm{kg}$, and muscle paralysis was obtained with rocuronium $0.6 \mathrm{mg} / \mathrm{kg}$. Anesthesia was maintained with $1.5-3.0$ vol\% sevoflurane and with a repeated dose of fentanyl 1.5-2.0 $\mu \mathrm{g} / \mathrm{kg}$. After endotracheal intubation, the lungs were ventilated with $50 \%$ oxygen with air. The ventilation was adjusted to an endexpiratory carbon dioxide pressure of 32-42 $\mathrm{mmHg}$. There was no induced hypotension and patients' blood pressure during the study was maintained in the normal range (systolic blood pressure $>100 \mathrm{mmHg}$ ). Before the surgical incision, the patients in the ulinastatin group were given 5,000 units/ $\mathrm{kg}$ of ulinastatin, which were mixed in $100 \mathrm{ml}$ normal saline intravenously over $30 \mathrm{~min}$, and the control group received the same volume of normal saline over the same duration. The patients and attending physicians were unaware of group allocations. All surgeries were performed using standardized surgical techniques. Maintenance fluid requirements were replaced with balanced crystalloid solutions at a dose of 4 $\mathrm{ml} / \mathrm{kg} / \mathrm{hr}$ and hydroxyethyl starch 6\% (Voluven ${ }^{\circledR}$, Fresenius Kabi, Germany) at a dose of $10 \mathrm{ml} / \mathrm{kg}$ with allowance for a maximum of $1,000 \mathrm{ml}$ as determined by the anesthetist according to central venous pressure readings, urine output, and clinical assessment. Units of packed red blood cells (pRBC) were transfused when hematocrit decreased below 28\% in patients who showed hypotension with low central venous pressure, or who showed hypotension and tachycardia, or who showed signs of severe anemia. The criteria for transfusing fresh frozen plasma (FFP) was INR $>1.5$, or PTT $\geq$ $1.5 \times$ normal baseline with continuing bleeding. The criteria for transfusing platelets were a platelet count $<50,000 /$ $\mathrm{mm} 3$, with continuing bleeding. The maintenance fluid and transfusion protocol were the same during and after surgery. 


\section{Measurements}

The primary endpoints were perioperative blood loss, blood transfusion, and fluid requirements. The amounts of infused fluid and transfused PRBC were recorded throughout the study period. The total operating time was also recorded. Intraoperative blood loss was recorded by measuring the volume in the suction apparatus and by estimating the amount of lost blood in the surgical pads. The apparent 12 h postoperative blood loss was determined by measuring the volume in the suction-drain bottles and blood in the dressing gauze. The secondary endpoints were changes of perioperative coagulation parameters. Three arterial blood samples were collected: before the induction of anesthesia (T1), at the end of surgery (T2), and $12 \mathrm{~h}$ after surgery (T3). From each sample, hemoglobin $(\mathrm{Hb})$ level and coagulation parameters including, platelet count, prothrombin time (PT), and activated partial thromboplastin time (aPTT) were measured immediately after collection. To measure AT III and FDP, the blood samples were centrifuged at 3,000 rpm for 20 min at $-4^{\circ} \mathrm{C}$. The plasma was stored in polypropylene tubes at $-80^{\circ} \mathrm{C}$ until measurement. FDP was measured using a latex agglutination method (Cobas Fara II; Roche, Switzerland). AT III activity was measured using a coagulometric test kit (Berichrom $^{\mathrm{TM}}$, Behring, Marburg, Germany).

\section{Statistics}

The primary outcome variable was to detect a difference in blood loss between the two groups. Since no previous information for blood loss was available at the time of planning the study, a sample size calculation was performed based on a pilot study. A reduction of $35 \%$ in blood loss in the ulinastatin group, compared with control group, was

Table 1. Demographic Data of the Patients

\begin{tabular}{lcc}
\hline & $\begin{array}{c}\text { Ulinastatin } \\
\text { group }\end{array}$ & $\begin{array}{c}\text { Control } \\
\text { group }\end{array}$ \\
\hline Age (yr) & $55 \pm 19.4$ & $52 \pm 14.9$ \\
Body weight (kg) & $62 \pm 11.1$ & $63 \pm 11.2$ \\
Sex (M/F) & $27 / 13$ & $22 / 18$ \\
ASA status: I/I//II & $18 / 20 / 2$ & $15 / 20 / 5$ \\
Surgery type (number of patients) & & \\
$\quad$ Spinal fusion & 17 & 17 \\
$\quad$ Number of fused vertebrae & $7 / 10$ & $14 / 3$ \\
$\quad: 1-2$ level/3-5 level & & \\
$\quad$ Total hip arthroplasty & 8 & 13 \\
Femur nailing & 13 & 2 \\
Fixation of pelvic bone fracture & 2 & $169 \pm 79.4$ \\
Duration of surgery (min) & $159 \pm 61.8$ &
\end{tabular}

Values are presented as number of patients or mean \pm SD. considered to be the smallest clinically significant difference. With the alpha level set to 0.05 and the power at 0.80, 38 patients are needed in each group. The data were analyzed using SPSS 15.0 (Windows Software Package, SPSS, Chicago, IL, USA). Demographic data between the groups were compared using the chi-square test, Fisher's exact test, or an independent t-test where appropriate. Data are expressed as the mean $\pm S D$. The Mann-Whitney U-test was used when the variables did not have a normal distribution. A P value of less than 0.05 was considered statistically significant.

\section{Result}

There were no differences in the characteristics of the patients and values measured preoperatively between the two groups (Table 1, 2). Total hip arthroplasty procedures were performed with cemented acetabular and femoral components.

\section{Primary endpoints}

The intraoperative blood loss was similar for both groups. However, $12 \mathrm{~h}$ postoperative blood loss was significantly lower in the ulinastatin group than in the control group (Table

Table 2. Perioperative Coagulation Data

\begin{tabular}{|c|c|c|c|}
\hline & & $\begin{array}{l}\text { Ulinastatin } \\
\text { group }\end{array}$ & $\begin{array}{l}\text { Control } \\
\text { group }\end{array}$ \\
\hline \multirow[t]{3}{*}{$\mathrm{Hb}(\mathrm{g} / \mathrm{dl})$} & $\mathrm{T} 1$ & $13 \pm 1.6$ & $13 \pm 2.0$ \\
\hline & $\mathrm{T} 2$ & $11 \pm 1.9^{*}$ & $11 \pm 1.6^{*}$ \\
\hline & $\mathrm{T} 3$ & $12 \pm 2.4^{*}$ & $11 \pm 1.9^{*}$ \\
\hline \multirow[t]{3}{*}{ PT (sec) } & $\mathrm{T} 1$ & $12 \pm 0.7$ & $12 \pm 0.7$ \\
\hline & $\mathrm{T} 2$ & $13 \pm 0.7^{*}$ & $13 \pm 1.0^{*}$ \\
\hline & T3 & $13 \pm 0.7^{*}$ & $13 \pm 1.0^{*}$ \\
\hline \multirow[t]{3}{*}{ aPTा (sec) } & $\mathrm{T} 1$ & $30 \pm 3.2$ & $29 \pm 3.2$ \\
\hline & $\mathrm{T} 2$ & $31 \pm 3.9$ & $30 \pm 2.6$ \\
\hline & T3 & $31 \pm 4.5$ & $30 \pm 4.7$ \\
\hline \multirow[t]{3}{*}{ Platelet count $\left(10^{3} / \mathrm{mm}^{3}\right)$} & $\mathrm{T} 1$ & $290 \pm 111.5$ & $305 \pm 150.9$ \\
\hline & $\mathrm{T} 2$ & $251 \pm 116.5$ & $269 \pm 146.2$ \\
\hline & T3 & $238 \pm 79.7$ & $261 \pm 139.9$ \\
\hline \multirow[t]{3}{*}{ Antithrombin III (\%) } & $\mathrm{T} 1$ & $89 \pm 15.9$ & $87 \pm 13.2$ \\
\hline & $\mathrm{T} 2$ & $76 \pm 15.5^{*}$ & $74 \pm 12.5^{*}$ \\
\hline & $\mathrm{T} 3$ & $86 \pm 15.9^{+}$ & $83 \pm 13.0^{+}$ \\
\hline \multirow[t]{3}{*}{ FDP (\%) } & $\mathrm{T} 1$ & $8 \pm 13.0$ & $10 \pm 13.1$ \\
\hline & $\mathrm{T} 2$ & $21 \pm 15.4^{*}$ & $25 \pm 26.6^{*}$ \\
\hline & T3 & $13 \pm 9.9^{\dagger}$ & $14 \pm 13.1^{\dagger}$ \\
\hline
\end{tabular}

Values are presented as mean \pm SD. T1: before surgery, T2: at the end of surgery, T3: $12 \mathrm{~h}$ after surgery, PT: prothrombin time, aPTT: activated partial thromboplastin time, $\mathrm{Hb}$ : hemoglobin, FDP: fibrinogen degradation product. ${ }^{*} P<0.05$ compared with the value at $\mathrm{T} 1 .{ }^{\dagger} \mathrm{P}<0.05$ compared with the value at $\mathrm{T} 2$. 
Table 3. Estimated Blood Loss, Transfused pRBC and Fluid Volume

\begin{tabular}{|c|c|c|c|}
\hline & & $\begin{array}{l}\text { Ulinastatin } \\
\text { group }\end{array}$ & $\begin{array}{l}\text { Control } \\
\text { group }\end{array}$ \\
\hline \multirow[t]{5}{*}{$\mathrm{T} 2$} & Total blood loss (ml) & $652.5 \pm 352.3$ & $667.5 \pm 325.3$ \\
\hline & $\begin{array}{l}\text { Estimated blood volume } \\
(\%)\end{array}$ & $15.0 \pm 7.9$ & $15.6 \pm 8.5$ \\
\hline & $\begin{array}{l}\text { Transfused pRBC volume } \\
(\mathrm{ml})\end{array}$ & $311.1 \pm 92.8$ & $400.0 \pm 194.4$ \\
\hline & Crystalloid (ml) & $1,560.0 \pm 749.0$ & $1,688.8 \pm 832.9$ \\
\hline & Colloid (ml) & $466.7 \pm 230.0$ & $486.7 \pm 50.8$ \\
\hline \multirow[t]{5}{*}{ T3 } & Total blood loss (ml) & $255.0 \pm 133.2^{*}$ & $395.4 \pm 338.4$ \\
\hline & $\begin{array}{l}\text { Estimated blood volume } \\
\text { (\%) }\end{array}$ & $6.0 \pm 3.4^{*}$ & $9.2 \pm 7.6$ \\
\hline & $\begin{array}{l}\text { Transfused pRBC volume } \\
\text { (ml) }\end{array}$ & $3,17.7 \pm 107.4$ & $294.2 \pm 121.9$ \\
\hline & Crystalloid (ml) & $1,578.8 \pm 605.1^{*}$ & $2,139.0 \pm 1073.7$ \\
\hline & Colloid (ml) & $3,71.4 \pm 75.6$ & $483.3 \pm 275.4$ \\
\hline
\end{tabular}

Values are presented as mean \pm SD or proportion. T2: at the end of surgery, T3: $12 \mathrm{~h}$ after surgery, pRBC: packed red blood cell. ${ }^{*} \mathrm{P}<0.05$ compared with control group.

3). The volume of crystalloid solution infused over the $12 \mathrm{~h}$ postoperative period was also lower in the ulinastatin group. The amounts of transfused pRBC throughout the study period did not differ between the two groups (Table 3). No patients required blood transfusion besides PRBC.

\section{Secondary endpoints}

$\mathrm{Hb}$ decreased and PT was prolonged at T2, without significant differences between the two groups (Table 2). Platelet count and aPTT did not change significantly during the study. In both groups, AT III levels were significantly lower at $\mathrm{T} 2$ than at $\mathrm{T} 1$, and this parameter recovered at $\mathrm{T} 3$ compared to T2 $(P<0.05)$. In both groups, FDP was significantly higher at $\mathrm{T} 2$ than at $\mathrm{T} 1$ and then decreased slightly at $\mathrm{T} 3$ compared to T2 $(P<0.05)$. AT III and FDP levels were not significantly different in the two groups.

\section{Discussion}

In this study, the administration of ulinastatin significantly reduced the early postoperative blood loss in patients with major orthopedic surgery. However, the administration of ulinastatin did not affect on the amounts of pRBC transfusion and coagulation parameters.

Ulinastatin (Ulistin ${ }^{\oplus}$, Han Lim Pharmaceutical, Seoul, Korea), a urinary trypsin inhibitor, is a glycoprotein derived from human urine [12]. It also has been called mingin, human inhibitor 30, miraclid, and bikunin [13]. Ulinastatin is indicated for acute inflammatory disorders, including acute pancreatitis, systemic inflammatory reaction syndrome, circulatory insufficiency, disseminated intravascular coagulation (DIC), and multiple organ failure [14].

Urinary trypsin inhibitor exists naturally in the human body and is secreted in greater quantities during stressful conditions, such as inflammation, cancer, infection, and tissue damage [15]. It is composed of 143 amino acid residues and two Kunitz-type protease inhibitor domains [16]. Both Kunitz domains inhibit serine proteases, especially elastase. The levels of proteases such as elastase, cathepsin $G$, and collagenase increase during infection and inflammation, and thus protease inhibitors have anti-inflammatory activity [17]. In addition, the second Kunitz domain may inhibit other enzymes such as coagulation factor Xa and plasma kallikrein [18]. Factor Xa plays a critical role at the start of the common pathway of the coagulation cascade [6]. Consequently, serine protease inhibitors downregulate coagulation and the fibrinolytic cascade $[15,19]$. Previous studies of ulinastatin have focused mainly on modulating inflammatory reactions. However, the hemostatic effects of ulinastatin in clinical practice have not been demonstrated clearly.

Orthopedic surgery involving the spine, pelvis, or femur can be associated with significant surgical and microvascular bleeding from venous sinuses due to difficulty in cauterization, increasing the need for blood product transfusions. However, allogenic blood transfusion carries the risks of infectious disease transmission and several noninfectious hazards, such as transfusion-related acute lung injury, post-transfusion purpura, transfusion-associated graft versus host disease, and transfusion-related immune modulation [20]. These risks have heightened the interest in blood conservation during surgery.

In the present study, a single infusion of ulinastatin reduced blood loss in the early postoperative period, suggesting that ulinastatin inhibits delayed bleeding. The coagulation parameters, including FDP and AT III, were similar in both groups throughout the study period. At the end of surgery, FDP was significantly higher, whereas AT III levels were significantly lower than before surgery in both groups. Because FDP is the product of the fibrinolysis cascade and AT III is consumed during hemorrhage, plasma FDP and AT III levels may be markers of coagulation status and the severity of hemorrhage [19]. Ulinastatin did not affect FDP or AT III, possibly because of the relatively low blood loss during the study period. No patients showed life threatening bleeding leading to coagulopathy.

PT was prolonged significantly, while aPTT remained unchanged in both groups, indicating that coagulation factors are consumed during hemorrhage but ulinastatin did not affect the consumption of these factors. Although PT and aPTT are influenced differently by the intrinsic and extrinsic coagulation pathways, there are abundant factors and coagulation components in the circulation. More enzymatic steps are involved in aPTT than in PT [21], which could explain the 
prolonged PT and no change in aPTT in the present study.

Various factors are involved in the coagulation cascade. For example, cytokines are primary mediators that cause an imbalance in the hemostatic system in DIC [7]. During the systemic inflammatory response, pro-inflammatory cytokines are produced from perturbed vascular endothelial cells and mononuclear cells, and this promotes intravascular fibrin formation and deposition [7]. Elevated levels of interleukin 6 and interleukin -8 are reduced by ulinastatin pretreatment in cardiac surgery [22]. Interleukin - 6 increases after trauma or surgical procedures and is positively correlated with the extent of tissue injury [23]. Peak circulating interleukin-6 occurs 4-6 hours after trauma, reflecting transcription time [24]. In humans, ulinastatin is excreted by the kidneys and its main function is probably in plasma [25]. The plasma halflife of ulinastatin during 0-3 hours after injection was 33 min and during the following 4 hours the half-life was 2 hours [25]. Thus, ulinastatin may inhibit inflammatory cytokines within a few hours of trauma and decrease early postoperative hemorrhage. However, further study of cytokine levels is required to confirm this hypothesis.

The beneficial effect of ulinastatin on blood loss under other clinical conditions, including sepsis, severe inflammatory states, bleeding and clotting disorders, is uncertain. The dose of ulinastatin and the timing of administration can also be limiting factors. To examine the perioperative effect of ulinastatin on inflammatory cytokines, ulinastatin was given at a dosage of 150,000 units every 12 hours from the start of induction of anesthesia until the fourth postoperative day [26]. Ulinastatin significantly modulated the acute phase response in the circulatory shock subsequent to hepatic resection. We used a lower dose because of its high cost to confirm its effect on blood loss. Nishiyama et al. [10] reported the effect on the fibrinolysis and coagulation during abdominal operation using intravenous administration of 6,000 units/ $\mathrm{kg}$ of ulinastatin. The single infusion of 5,000 units $/ \mathrm{kg}$ of ulinastatin in our study showed reduced postoperative blood loss in patients receiving orthopedic surgery. We also did not strictly perform a protocol for blood transfusion in the postoperative period. The amounts of transfused pRBC that patients received in the postoperative period were dependent on an assessment of clinical needs, symptoms, and surgeons' preference rather than our protocol alone. However, staffs were blinded to the treatment group, limiting the ability to bias the results. These results also require further work to identify a dose-dependent effect of ulinastatin on blood loss.

In conclusion, a single infusion of ulinastatin during major orthopedic surgery reduces blood loss in the early postoperative period. Ulinastatin may be useful when continuous postoperative bleeding from venous sinuses is expected. The potential beneficial effects of cytokines in perioperative blood loss with the mechanism of action deserve further investigation.

\section{Acknowledgements}

This study was supported by research funds from the Catholic University Medical Center, 2008. We disclose that the paper has no financial conflict of interest.

\section{References}

1. Guay j, Reinberg C, Poitras B, David M, Mathews S, Lortie $L$, et al. A trial of desmopressin to reduce blood loss in patients undergoing spinal fusion for idiopathic scoliosis. Anesth Analg 1992; 75: 405-10.

2. Mylod AG Jr, France MP, Muser DE, Parsons JR. Perioperative blood loss associated with total knee arthroplasty. A comparison of procedures performed with and without cementing. J Bone Joint Surg Am 1990; 72: 1010-2.

3. Levy JH, Sypniewski E. Aprotinin: a pharmacologic overview. Orthopedics 2004; 27: s653-8.

4. Ng KF, Lo JW. The development of hypercoagulability state, as measured by thromboelastography, associated with intraoperative surgical blood loss. Anaesth Intensive Care 1996; 24: 20-5.

5. Stokke T, Burchardi H, Hensel I, Kostering H, Kathner T, Rahlf $\mathrm{G}$. Continuous intravenous infusion of elastase in normal and agranulocytic minipigs-effects on the lungs and the blood coagulation system. Resuscitation 1986; 14: 61-79.

6. Bauer KA. New anticoagulants. Curr Opin Hematol 2008; 15: 509-15.

7. Levi M. Disseminated intravascular coagulation. Crit Care Med 2007; 35: 2191-5.

8. Okida M, Masako O, Maruya H, Higashi T, Yukaya H. Intraoperative changes in blood coagulation and the effectiveness of ulinastatin during liver resection. J Anesth 1991; 5: 43-7.

9. Porte RJ, Molenaar IQ, Begliomini B, Groenland TH, Januszkiewicz A. Aprotinin and transfusion requirements in orthotopic liver transplantation: a multicentre randomized double-blind study. EMSALT Study Group. Lancet 2000; 355: 1303-9.

10. Nishiyama T, Yokoyama T, Yamashita K. Effects of a protease inhibitor, ulinastatin, on coagulation and fibrinolysis in abdominal surgery. J Anesth 2006; 20: 179-82.

11. Baudo F, DeGasperi A, deCataldo F, Caimi TM, Cattaneo D, Redaelli R, et al. Antithrombin III supplementation during orthotopic liver transplantation in cirrhotic patients: a randomized trial. Thromb Res 1992; 68: 409-16.

12. Hirose J, Ozawa T, Miura T, Isaji M, Nagao Y, Yamashiro K, 
et al. Human neutrophil elastase degrades inter-alphatrypsin inhibitor to liberate urinary trypsin inhibitor related proteins. Biol Pharm Bull 1998; 21: 651-6.

13. Pugia MJ, Lott JA. Pathophysiology and diagnostic value of urinary trypsin inhibitors. Clin Chem Lab Med 2005; 43: $1-16$.

14. Inoue K, Takano H, Yanagisawa R, Yoshikawa T. Protective effects of urinary trypsin inhibitor on systemic inflammatory response induced by lipopolysaccharide. J Clin Biochem Nutr 2008; 43: 139-42.

15. Pugia MJ, Valdes R Jr, Jortani SA. Bikunin (urinary trypsin inhibitor): structure, biological relevance, and measurement. Adv Clin Chem 2007; 44: 223-45.

16. Kobayashi H, Suzuki M, Hirashima Y, Terao T. The protease inhibitor bikunin, a novel anti-metastatic agent. Biol Chem 2003; 384: 749-54.

17. Mania-Pramanik J, Potdar SS, Vadigoppula A, Sawant S. Elastase: a predictive marker of inflammation and/or infection. J Clin Lab Anal 2004; 18: 153-8.

18. Nii A, Morishita H, Yamakawa T, Matsusue T, Hirose J, Miura $T$, et al. Design of variants of the second domain of urinary trypsin inhibitor (R-020) with increased factor Xa inhibitory activity. J Biochem 1994; 115: 1107-12.

19. Nesheim M. Thrombin and fibrinolysis. Chest 2003; 124(3 Suppl): 33S-9S.

20. Hendrickson JE, Hillyer CD. Noninfectious serious hazards of transfusion. Anesth Analg 2009; 108: 759-69.

21. Martini WZ, Cortez DS, Dubick MA, Park MS, Holcomb JB. Thromboelastography is better than PT, aPTT, and activated clotting time in detecting clinically relevant clotting abnormalities after hypothermia, hemorrhagic shock and resuscitation in pigs. J Trauma 2008; 65: 535-43.

22. Kawamura T, Inada K, Akasaka N, Wakusawa R. Ulinastatin reduces elevation of cytokines and soluble adhesion molecules during cardiac surgery. Can J Anaesth 1996; 43: 456-60.

23. Pullicino EA, Carli F, Poole S, Rafferty B, Malik ST, Elia M. The relationship between the circulating concentrations of interleukin 6 (IL - 6), tumor necrosis factor (TNF) and the acute phase response to elective surgery and accidental injury. Lymphokine Res 1990; 9: 231-8.

24. Cruickshank AM, Fraser WD, Burns HJ, Van Damme J, Shenkin A. Response of serum interleukin - 6 in patients undergoing elective surgery of varying severity. Clin Sci (Lond) 1990; 79: 161-5.

25. Jonsson-Berling BM, Ohlsson K. Distribution and elimination of intravenously injected urinary trypsin inhibitor. Scand J Clin Lab Invest 1991; 51: 549-57.

26. Ambiru S, Miyazaki M, Sasada K, Ito H, Kimura F, Nakagawa $K$, et al. Effects of perioperative protease inhibitor on inflammatory cytokines and acute-phase proteins in patients with hepatic resection. Dig Surg 2000; 17: 337-43. 